\title{
Sensorimotor impairments, postural instability and risk of falling in older adults with Diabetic Peripheral Neuropathy
}

\author{
AH Wettasinghe ${ }^{\text {a\# }}$, DWN Dissanayake ${ }^{\mathrm{b}}$, L Allet $^{\mathrm{c}, \mathrm{d}}$, P Katulanda $^{\mathrm{e}}$, SR Lord $^{\mathrm{f}}$ \\ a Department of Allied Health Sciences, Faculty of Medicine, University of Colombo, Sri Lanka \\ bepartment of Physiology, Faculty of Medicine, University of Colombo, Sri Lanka \\ 'Department of Community Medicine, University Hospitals and University of Geneva, Geneva, Switzerland \\ ${ }^{\mathrm{d}}$ Department of Physiotherapy, School for Health Sciences, HES-SO, University of Applied Sciences \& \\ Arts of Western Switzerland, Geneva, Switzerland \\ e Department of Clinical Medicine, Faculty of Medicine, University of Colombo, Sri Lanka \\ fNeuroscience Research Australia, UNSW, Randwick, Sydney, Australia \\ \#for correspondence; \\ E-mail- asha@med.cmb.ac.lk \\ Telephone numbers- +94777822193, +94112688686 \\ Postal address- 25 Kynsey Rd, Colombo 00800, Department of Allied Health Sciences, Faculty of \\ Medicine, University of Colombo, Sri Lanka
}




\begin{abstract}
Background-Older adults with type-2 diabetes mellitus (DM) have high incidence of falls. The aim of this study was to compare sensorimotor functions balance, mobility, fear of falling and fall history in older people with DM (with and without neuropathy) and non-diabetic healthy controls.
\end{abstract}

Methods-We enrolled 153 participants aged 50-70years; 51 people with diabetic peripheral neuropathy (D-PN), 52 with diabetes without neuropathy (D-noPN) and 50 healthy controls (HC). Participants completed a fear of falling assessment and detailed test battery comprising sensorimotor functions, lower limb strength, contrast vision, reaction time, balance and mobility from which a composite physiological fall risk score was derived (PFRS). In addition, a fall history of the past three months was recorded.

Results-Post-hoc comparisons of ANOVA test revealed the D-PN had significant deficits than the other two groups in tests of lower limb sensation, knee extension strength, reaction time, postural sway, one leg standing, sit-to-stand and the timed up and go test. The D-PN had the highest fear of falling (30.18 \pm 6.75$)$ and the highest PFRS (1.68 \pm 1.13$)$. PFRS for the D-noPN $(0.74 \pm 0.80)$ were intermediate between HC $(0.49 \pm 0.96)$ and DP-N groups. Thirty-four D-PN participants $(66.7 \%), 19 \mathrm{D}-$ noPN participants $(36.5 \%)$ and $7 \mathrm{HC}(14.0 \%)$ reported one or more falls in the past three months; Chi2 test for trend $=28.1, \mathrm{df}=2, \mathrm{p}<0.001$ ).

Conclusions-Older people with diabetic neuropathy have impaired sensorimotor function, balance, mobility, and associated increased fear of falling and fall rates. This population may benefit from fall risk assessments involving the above measures, and subsequent interventions targeted to deficits amenable to correction.

Keywords postural control; sensorimotor functions; diabetic neuropathy; fall risk

\title{
1. Introduction
}

Diabetes mellitus (DM) affects approximately $8.5 \%$ of people aged over 18 years [1] and more than $16 \%$ of people aged over 65 years [2]. With changing demography and higher rates of diagnosis, the prevalence of DM is expected to increase from 366 million in 2011 to 553 million in 2030 [3]. DM was previously considered as a disease of the developed world, but has now become a global epidemic, with two thirds of the world diabetic population living in the developing countries [4]. Sri Lanka is no exception: with prevalence studies undertaken over the past 30 years showing a relentless upward surge of DM in both urban and rural districts. In consequence, DM is having major impacts on sufferers and the health care system. A study carried out in 2005 in adults aged between 35 and 65 years in four provinces in Sri Lanka reported the prevalence of DM as $14.2 \%$ in men and $13.5 \%$ in women [5]. According to Sri Lanka Diabetes and Cardiovascular Study (SLDCS) which was carried out in the same year (i.e. 2005), the national prevalence of diabetes mellitus in Sri Lanka was $10.3 \%$ [6]. The SLDCS also reported that one in five adults in Sri Lanka has either diabetes or pre-diabetes and onethird of those with diabetes were undiagnosed [6].

Diabetic peripheral neuropathy (D-PN) is a common and costly chronic complication and a major factor that reduces mobility and quality of life of DM patients [7]. D-PN affects up to 50\% of people with DM and usually starts with damage to the peripheral sensory nerves which progresses later to motor and autonomic nerves $[8,9]$. National prevalence of D-PN in 2012, according to diabetic neuropathy symptom score among all patients, patients with already established diabetes and newly diagnosed patients were $48.1 \%, 59.1 \%$ and $28.8 \%$ respectively [10]. Diabetic neuropathy causes progressive loss of proprioception, reflexes and lower limb strength which lead to increased postural instability [11]. Studies have reported a significant association between D-PN severity and fall risk $[12,13]$ with one study reporting that people with DPN are 15 times more likely to experience a fall compared to healthy controls [14].

Risk factors in DM, and particularly for those with neuropathy, extend well beyond sensory loss. Previous studies have found that in addition to sensory loss and advanced disease status [15], muscle weakness [16], increased postural sway [17], gait and mobility impairments [18, 19] and fear of falling [15] increase fall risk in people with DM. Further, older people with DM have increased risks of frailty and osteoporosis with subsequent increased morbidity and mortality associated with fall-related fractures [20-23]. Therefore fall prevention is a high priority for this population. However, previous studies have used only a limited range of tests to assess potential risk factors for falls, and many have used expensive high tech equipment that is unavailable in most clinical settings throughout the world. 
Therefore, the aim of this cross sectional comparative study was to compare a broad set of sensorimotor, balance and functional mobility measures in community-living people with DM (with and without diagnosed peripheral neuropathy) and people without DM. We hypothesized that those with DM would have multiple physiological and functional impairments and significantly increased composite physiological fall risk scores, and this would be particularly the case for those with diabetic peripheral neuropathy. The study findings may elucidate explanatory and modifiable risk factors for falls in people with DM.

\section{Materials and Methods}

\subsection{Participants}

One hundred fifty three participants $(n=153$; females=68) comprised the study sample: 51 with diabetic peripheral neuropathy (D-PN group), 52 with non-neuropathic diabetes (D-noPN group) and 50 without diabetes (healthy control (HC) group). The participants with diabetes were recruited from Endocrinology clinics at the National Hospital, Colombo, Sri Lanka. The 50 HC participants were also recruited from the National Hospital (hospital and university staff, non-diabetic patients) and through community sources. Assessments were performed in the Neurophysiology laboratory at the Faculty of Medicine, University of Colombo. We based our sample size calculation on detecting differences in the proportion of fallers among the groups. This revealed 47 participants per group were required to detect as significant at the 5\% level (one-sided) and $80 \%$ power, twice the proportion of fallers in the DM groups (i.e. 50\%) compared with the HC group (i.e. 25\%).

An endocrinologist examined all participants with diabetes. Peripheral neuropathy was confirmed using validated symptom scores and examination scores for D-PN - see neuropathy assessment below in 2.3.1. HbA1c tests were used to assess glycemic control in the D-PN and D-noPN groups and exclude undiagnosed diabetes in the HC group.

Inclusion criteria for the diabetes groups included having diabetes more than five years, aged 50-70 years, living in the community, being able to understand instructions and able to ambulate household distances without an assistive device. Participants in all groups with significant central nervous system dysfunctions, musculoskeletal deformity or lower limb pathologies that affect balance were excluded.

Ethics approval was obtained from the Ethics Review Committee of the Faculty of Medicine, University of Colombo, Sri Lanka and from the National Hospital Colombo, Sri Lanka. Informed verbal and written consent was obtained from all the participants prior to study participation. The study was designed and conducted in compliance with the Declaration of Helsinki.

\section{a. Anthropometric, demographic and medical measures}

Anthropometric, demographic and medical information were obtained by means of an interviewer administered questionnaire and clinical examination. Fear of falling was assessed with the Icon-FES [24]. The shortened version of Icon-FES scale is an interview-based questionnaire using a combination of pictures and matching short phrases. Icon-FES provides information on level of concern about falls for a range of activities of daily living. The shortened Icon-FES version contains 10 items scored on a 4-point scale $(1=$ not at all concerned to 4 = very concerned).

\section{b. Physical assessments}

\section{i. Neuropathy and lower limb sensation}

Diabetic Neuropathy Symptom (DNS) [25] and Diabetic Neuropathy Examination (DNE) [25] scores were used for diagnosing diabetic polyneuropathy. The DNS score comprises four items: symptoms of unsteadiness in walking, neuropathic pain, paraesthesia and numbness. Each symptom is scored as 1 point with a score of 1 is sufficient to indicate D-PN. The DNE score contains two items concerning muscle strength, one concerning reflexes and five concerning sensation. Each item is scored from 0 (normal) to 2 (2 severely disturbed). The maximum score is 16 points, and a score $>3$ points is indicative of D-PN.

Nerve conduction studies of tibial and sural nerve velocity, amplitude and latency values were assessed by a neurophysiologist using a Natus Xltek nerve conduction device (Koll Center Parkway Suite, Pleasanton, CA, 
USA) [25]. Vibration perception thresholds of the big toe were measured using a Biothesiometer (Bio Medical Instrument co, Ohio, USA) [25].

Tactile sensitivity was assessed with a Semmes-Weinstein pressure aesthesiometer comprising 20 nylon filaments of equal length with varying diameter [25]. The filaments were applied to the center of the lateral malleolus, and pressure measurements were expressed as logarithms of the bending force in milligrams. Lower limb proprioception was measured using a lower limb matching task with participants sitting and eyes closed. Errors in matching the great toes were recorded using a protractor inscribed on a vertical clear acrylic sheet $(60 \times 60 \times 1 \mathrm{~cm})$ placed between the legs [26].

\section{ii. Muscle strength}

Maximal isometric quadriceps strength was measured in both legs while participants were seated on a high chair (so that feet did not touch the floor) with the hips and knees flexed to $90^{\circ}$ [26]. A strain gauge was fixed horizontally with straps on the lower shin, after which the participant was given three attempts with the dominant leg to push against the strap as forcefully as possible. In addition, the five time sit to stand test was administered [27]. Participants were given a test trial and then the second trial was taken as the test result.

\section{iii. Balance and Gait}

Postural sway was assessed using a sway meter that measured displacements of the body at the level of the waist. Testing was performed with participants standing on the floor and on a foam rubber mat $(40 \times 40 \times 15 \mathrm{~cm}$ thick) with eyes open and closed [26]. Sway path (number of mm squares traversed by the sway meter pen) for each $30 \mathrm{~s}$ test was recorded. Standing balance was also assessed by timing how long participants could stand on one leg maximum of 30 seconds [24]. Functional mobility was assessed with the Timed Up and Go (TUG) test [29]. Participants were asked to rise from a chair, walk forward three meters at their usual walking pace, turn 180 degrees, walk back to the chair and sit down. The TUG time was obtained by timing how long a participant took to complete the whole process.

\section{iv. Contrast vision and reaction time}

Visual contrast sensitivity was assessed using the Melbourne Edge Test, which presents 20 circular patches containing edges with reducing contrast [26]. Correct identification of the orientation of the edge on the patches provides a measure of contrast sensitivity in decibel units, where $1 \mathrm{~dB}=10 \log 10$ contrast. Simple reaction time in milliseconds was assessed using a light as a stimulus and a button press as the response [26].

\section{v. Physiological fall risk}

Physiological fall risk was estimated using the Physiological Profile Assessment (PPA) [26] which comprises five of the above physical tests evaluating key functions of the human balance system: lower limb proprioception, visual contrast sensitivity, knee extension strength, simple reaction time and postural sway when standing on a compliant surface [25, 26]. The five PPA components were weighted to compute a composite PPA fall risk score expressed in standard (z-score) units, with high scores indicating poorer physical performance. In multivariate models, weighted contributions from these five variables provide a fall risk score that can predict community-dwelling older Caucasian people at risk of multiple falling over a 12 -month period with $75 \%$ accuracy [30].

\section{c. Falls}

Falls were defined as unexpected events which resulted in the participant unintentionally coming to the ground, floor or other lower level [31]. Participants who reported one or more falls in the past three months were defined as fallers.

\section{d. Statistical analysis}

Variables with right skewed distributions (tibial nerve velocity, tactile sensitivity, five time sit to stand time, sway, one leg stand tests and TUG time) were normalized using log transformations. One-way ANOVAs with Turkey post-hoc test between group comparisons were used in all analyses. Falls status was assessed with the $\mathrm{Chi}^{2}$ test for trend. A $5 \%$ limit of significance was applied. Analyses were conducted using SPSS for windows 
(IBM SPSS Statistics for Windows, Version 22.0. Armonk, NY: IBM Corp.) and Epi Info (Centers for Disease Control and Prevention in Atlanta, Georgia).

\section{Results}

\subsection{Anthropometric, demographic and medical measures}

The D-PN, D-noPN and HC groups were well matched for age, height and weight. The D-PN group had longer disease duration than the D-noPN group, and both diabetes groups were taking significantly more medications than the HC group (Table 1). Twenty six D-PN participants (51\%) and five D-noPN participants $(9.6 \%)$ were insulin-dependent.

\section{a. Lower limb sensation}

There were significant differences among the three groups for all four lower limb sensation tests: tibial nerve conduction velocity $(P<0.001)$, vibration perceptual thresholds $(P<0.001)$, tactile sensitivity $(P<0.001)$ and proprioception $(P<0.001)$. Post-hoc comparisons indicated both diabetes groups had significantly poorer scores in all tests compared the HC group, and that the D-PN group had poorer scores than the D-noPN group in the vibration perceptual threshold and tactile sensitivity tests (Table 1). D-noPN group also have significantly poorer scores than $\mathrm{HC}$ in tibial nerve velocity, vibration perception threshold, proprioception and tactile sensitivity (Table 1).

\section{b. Muscle strength, Balance, Gait and Reaction time}

There were significant differences among the three groups for the simple reaction time $(P=0.001)$, knee extension strength $(P<0.001)$, five time sit to stand $(P<0.001)$ (Table 2$)$, one leg stand $(P<0.001)$, timed up and go $(P<0.001)$, sway on foam eyes open and eyes closed tests (Table 3$)$. Post-hoc comparisons indicated both diabetes groups had significantly slower five time sit to stand times compared the HC group, and that the D-PN group had poorer scores than both the D-noPN and HC groups in the simple reaction time, knee extension strength, one leg stand, timed up and go, and sway on foam eyes open and eyes closed tests (Tables 2 and 3).

\section{c. Falls, physiological fall risk and fear of falling}

Thirty four D-PN participants (66.7\%), 19 D-noPN participants (36.5\%) and 7 HC (14.0\%) reported one or more falls in the past three months; $\mathrm{Chi}^{2}$ test for trend $=28.1, \mathrm{df}=2, P<0.001$ ).

Compared with the HC group, the D-noPN and D-PN groups had markedly increased odds of being a faller: 3.54 and 12.3 increased odds respectively. Mean PPA and Icon-FES scores differed significantly among the groups in the ANOVA, and post hoc analyses revealed that the D-PN group had higher PPA scores than both the $\mathrm{HC}$ and D-noPN groups and that Icon-FES scores differed significantly between each group. The PPA fall risk profiles for the D-PN and D-noPN groups are presented in Figure 1. These profiles indicate that the D-noPN groups had a similar profile to the reference group of people without diabetes aged 65 years and over, whereas the D-PN group had below average scores in the tests of proprioception, knee extension strength, reaction time and postural sway.

\section{Discussion}

In this study, we found that sensorimotor, balance and functional mobility measures were impaired in older people with DM and this more so the case in people with diabetic neuropathy. Specifically, we found that those with diabetic neuropathy had significantly poorer knee extension strength, reaction time, standing balance and mobility in addition to lower limb sensory loss. Such deficits across a diverse range of neuromuscular systems provide insight into why older people with DM are at increased risk of falls.

As expected, the D-PN group exhibited significant loss in all lower limb tests important for sensory integrity: tibial nerve conduction velocity, vibration perceptual thresholds, tactile sensitivity and proprioception. However, while the D-noPN group did not have manifested neuropathy they also had significantly impaired sensory loss 
in all four sensory measures, compared with the healthy controls. This indicates that there is significant subclinical sensory loss in people with diabetes without manifested neuropathy that may warrant further assessment and management. These findings align with previous work that has reported impairments in proprioception and lack of sensory feedback cueing contribute to reduced postural control $[32,33]$ and impair balance recovery when people are exposed to unexpected perturbations [34]. Further deleterious effects are evident in people with D-PN adopting a rigid postural control strategy once they perceive increased sway. This stiffening strategy is associated with level of neuropathy and history of DM [32].

In addition to sensory loss, previous studies have documented that people with type $2 \mathrm{DM}$ have reduced muscle mass [35-37], poor functional strength, balance and gait impairments [38]. It has been shown that postural sway is increased in people with D-PN [39-41] and especially in eye closure test conditions [32, 42]. Some studies have reported the postural sway with eyes open in older people with D-PN is comparable to postural sway with eyes closed of healthy adults $[34,40]$. Further, D-PN patients tend to use a hip strategy rather than an ankle strategy for balance tests when they are deprived of visual inputs [43]. We also found that older people with DM have significant impairments in the physiological domains of strength, reaction time, and balance. It is likely that this impaired mobility as assessed with the TUG test results from impairments to multiple sensorimotor and balance domains, as it has been shown these underpin this functional mobility measure [44].

DPN group has increased risk of falls compared to other groups. This elevated risk aligns with previous studies $[12,13,45]$ that have been conducted both retrospectively [13] and prospectively [45]. Increased fall risk was associated with concomitant increases in concern about falling and physiological fall risk. The PPA profiles show the D-noPN group had similar test scores to a significantly older (i.e. 65+ years) reference group without diabetes and the D-PN group had scores below these reference levels indicating impaired functioning (Figure 1). Further, the average composite PPA score for the D-PN group of 1.68 indicates a marked risk of falls based on population norms [46].

Strengths of this study include the setting of the study in a developing country in which DM is a serious health issue, the inclusion of a broad range of sensorimotor and balance measures and the recruitment of large samples of older community-living people with DM with and without peripheral neuropathy along with a well matched group of healthy controls. We also acknowledge certain limitations. First, we did not include measures of executive functioning such as cognition or mood as part of the test battery; factors implicated with fall risk in older people and clinical groups with balance impairment [47]. Second, falls were ascertained retrospectively. Further studies with large samples and prospective follow-up for falls are required to develop definitive fall risk profiles for people with DM.

Our findings reveal that older people with DM, with and without neuropathy, have multiple sensorimotor, balance and mobility impairments; many of which are amenable to intervention. Most of the assessments used here comprise simple "low-tech" tests with scope for widespread use in clinical settings. The identification of specific impairments would aid greatly in diabetic management, through education, medication prescription, and lifestyle modification comprising healthy diets and regular exercise containing balance training. The findings may facilitate fall prevention initiatives by assisting in the identification of individuals at increased risk of falls and the initiation of targeted interventions for preventing falls and fractures.

In conclusion, the study findings indicate that older people with DM have impaired sensorimotor function, balance and mobility, and associated increased fear of falling and fall rates. Older people with DM, and especially those with neuropathy, would benefit from fall risk assessments involving the above measures, and subsequent interventions targeted to deficits amenable to correction.

\section{Compliance with ethical standards}

This study was approved by the Ethical Review Committee of the University of Colombo (EC-15-166) and the National Hospital of Sri Lanka, Research Ethics Committee (ETH/COM/2016). All participants signed informed consent prior to their participation.

\section{Conflict of Interest}

The PPA (NeuRA FallScreen) is commercially available through Neuroscience Research Australia. 


\section{Acknowledgments}

We wish to thank the management of National Hospital Colombo, Sri Lanka and Faculty of Medicine, University of Colombo, Sri Lanka for ethics approval and facilities provided for this study to make this research successful.

\section{Funding}

This work was supported by the Postgraduate research Scholarship (AP/3/2/2016/PG/05), University of Colombo, Sri Lanka.

\section{Contributors}

All authors contributed equally in conceptualization of the study. Data collection was conducted by AHW under direct guidance and supervision of PK and DWND. AHW was trained for the methods of the study by PK, DWND and SL. AHW prepared the manuscript under the guidance of DWND and SL. SL and DWND edited the manuscript. LA and PK contributed to the manuscript by editing and reviewing the manuscript. Final manuscript was reviewed and approved by all the authors for submission.

\section{References}

1. World Health Organization. Diabetes fact sheet. 2019. https://www.who.int/news-room/factsheets/detail/diabetes. Accessed 15 May 2019.

2. Sánchez Martínez M, Blanco A, Castell MV, Gutiérrez Misis A, González Montalvo JI, Zunzunegui MV, et al. Diabetes in older people: Prevalence, incidence and its association with medium- and longterm mortality from all causes. Aten Primaria. 2014;46(7):376-84.

3. International Diabetes Federation, Diabetes Atlas:The global burden. 5th ed. 2011 https://www.idf.org/e-library/epidemiology-research/diabetes-atlas/20-atlas-5th-edition.html. Accessed 11 May 2019

4. Katulanda P, Sheriff MH, Matthews DR. The diabetes epidemic in Sri Lanka - a growing problem. Ceylon Med J. 2006;51(1):26-8.

5. Wijewardene K, Mohideen MR, Mendis S, Fernando DS, Kulathilaka T, Weerasekara D, Uluwitta P, et al. Prevalence of hypertension, diabetes and obesity: baseline findings of a population based survey in four provinces in Sri Lanka. Ceylon Med J. 2005;50(2):62-70.

6. Katulanda P, Constantine GR, Mahesh JG, Sheriff R, Seneviratne RD, Wijeratne S, et al. Prevalence and projections of diabetes and pre-diabetes in adults in Sri Lanka--Sri Lanka Diabetes, Cardiovascular Study (SLDCS). Diabet Med. 2008;25(9):1062-9.

7. Deli G, Bosnyak E, Pusch G, Komoly S, Feher G. Diabetic neuropathies: diagnosis and management. Neuroendocrinology. 2013;98(4):267-80.

8. Tesfaye S, Selvarajah D. Advances in the epidemiology, pathogenesis and management of diabetic peripheral neuropathy. Diabetes Metab Res Rev. 2012;28 Suppl 1:8-14.

9. Kaur S, Pandhi P, Dutta P. Painful diabetic neuropathy: an update. Ann Neurosci. 2011;18(4):168-75.

10. Katulanda P, Ranasinghe P, Jayawardena R, Constantine GR, Sheriff MH, Matthews DR. The prevalence, patterns and predictors of diabetic peripheral neuropathy in a developing country. Diabetol Metab Syndr. 2012;4(1):21.

11. Ites KI, Anderson EJ, Cahill ML, Kearney JA, Post EC, Gilchrist LS. Balance interventions for diabetic peripheral neuropathy: a systematic review. J Geriatr Phys Ther. 2011;34(3):109-16.

12. Richardson JK, Ching C, Hurvitz EA. The relationship between electromyographically documented peripheral neuropathy and falls. J Am Geriatr Soc. 1992;40(10):1008-12.

13. Richardson JK, Hurvitz EA. Peripheral neuropathy: a true risk factor for falls. J Gerontol A Biol Sci Med Sci. 1995;50(4):M211-5.

14. Dingwell JB, Ulbrecht JS, Boch J, Becker MB, O'Gorman JT, Cavanagh PR. Neuropathic gait shows only trends towards increased variability of sagittal plane kinematics during treadmill locomotion. Gait Posture. 1999;10(1):21-9.

15. Bokan-Mirković V, Škarić-Karanikić Ž, Nejkov S, Vuković M, Ćirović D. Diabetic Polyneuropathy and Risk of Falls: Fear of Falling and Other Factors. Acta Clin Croat. 2017;56(4):721-727.

16. Macgilchrist C, Paul L, Ellis BM, Howe TE, Kennon B, Godwin J. Lower-limb risk factors for falls in people with diabetes mellitus. Diabet Med, 2010;27(2):162-8.

17. Dixit S, Maiya A, Shasthry BA, Kumaran DS, Guddattu V. Postural sway in diabetic peripheral neuropathy among Indian elderly. Indian J Med Res. 2015;142(6):713-20.

18. Mustapa A, Justine M, Mustafah NM, Jamil N, Manaf H. Postural Control and Gait Performance in the Diabetic Peripheral Neuropathy: A Systematic Review. BioMed Research International. 2016:14. 
19. Alvarenga PP, Pereira DS, Anjos DM. Functional mobility and executive function in elderly diabetics and non-diabetics. Rev Bras Fisioter. 2010;14(6):491-6.

20. Jackuliak P, Payer J. Osteoporosis, Fractures, and Diabetes. International Journal of Endocrinology. 2014:820615.

21. Paschou SA, Dede AD, Anagnostis PG, Vryonidou A, Morganstein D, Goulis DG. Type 2 Diabetes and Osteoporosis: A Guide to Optimal Management. The Journal of Clinical Endocrinology \& Metabolism. 2017;102(10):3621-3634.

22. Lipscombe LL, Jamal SA, Booth GL, Hawker GA. The Risk of Hip Fractures in Older Individuals With Diabetes. A population-based study. Diabetes Care. 2007;30(4):835-841.

23. Cobo A, Vázquez LA, Reviriego J, Rodríguez-Mañas L. Impact of frailty in older patients with diabetes mellitus: An overview. Endocrinol Nutr. 2016;63(6):291-303.

24. Henderson EJ, Lord SR, Bordie MA, Gaut DM, Lawrence AD, Close TCT, et al. Rivastigmine for gait stability in patients with Parkinson's disease (ReSPonD): a randomised, double-blind, placebocontrolled, phase 2 trial. Lancet Neurol. 2016;15(3):249-58.

25. Mythili A, Kumar KD, Subrahmanyam KA, Venkateswarlu K, Butchi RG. A Comparative study of examination scores and quantitative sensory testing in diagnosis of diabetic polyneuropathy. Int $\mathbf{J}$ Diabetes Dev Ctries. 2010;30(1):43-8.

26. Lord SR, Menz HB, Tiedemann A. A physiological profile approach to falls risk assessment and prevention. Phys Ther. 2003;83(3):237-52.

27. Makizako H, Shimada H, Doi T, Tsutsumimoto K, Nakakubo S, Hotta R, Suzuki T. Predictive Cutoff Values of the Five-Times Sit-to-Stand Test and the Timed "Up \& Go" Test for Disability Incidence in Older People Dwelling in the Community. Phys Ther. 2017;97(4):417-424.

28. Allet L, Kim H, Ashton-Miller J, De Mott T, Richardson JK. Frontal plane hip and ankle sensorimotor function, not age, predicts unipedal stance time. Muscle Nerve. 2012;45(4):578-85.

29. Barry E, Galvin R, Keogh C, Horgan F, Fahey T. Is the Timed Up and Go test a useful predictor of risk of falls in community dwelling older adults: a systematic review and meta-analysis. BMC Geriatr. 2014;14:14.

30. Lord SR, Ward JA. Age-associated differences in sensori-motor function and balance in community dwelling women. Age Ageing. 1994;23(6):452-60.

31. Lamb SE, Jørstad-Stein EC, Hauer K, Becker C. Development of a common outcome data set for fall injury prevention trials: the Prevention of Falls Network Europe consensus. J Am Geriatr Soc. 2005;53(9):1618-22.

32. Toosizadeh N, Mohler J, Armstrong DG, Talal TK, Najafi B. The influence of diabetic peripheral neuropathy on local postural muscle and central sensory feedback balance control. PLoS One. 2015;10(8):e0135255.

33. Kars HJ, Hijmans JM, Geertzen JH, Zijlstra W. The effect of reduced somatosensation on standing balance: a systematic review. J Diabetes Sci Technol. 2009;3(4):931-43.

34. Lafond D, Corriveau H, Prince F. Postural control mechanisms during quiet standing in patients with diabetic sensory neuropathy. Diabetes Care. 2004;27(1):173-8.

35. Kim TN, Park MS, Yang SJ, Yoo HJ, Kang HJ, Song W, et al. Prevalence and Determinant Factors of Sarcopenia in Patients With Type 2 Diabetes. The Korean Sarcopenic Obesity Study (KSOS). 2010;33(7):1497-1499.

36. Wang T, Feng X, Zhou j, Gong H, Xia s, Wei Q, et al. Type 2 diabetes mellitus is associated with increased risks of sarcopenia and pre-sarcopenia in Chinese elderly. Sci Rep. 2016;6:38937.

37. Park SW, Goodpaster BH, Strotmeyer ES, de Rekeneire N, Harris TB, Schwartz AV, et al. Decreased muscle strength and quality in older adults with type 2 diabetes: the health, aging, and body composition study. Diabetes. 2006;55(6):1813-8.

38. Vaz MM, Costa GC, Reis JG, Junior WM, Albuquerque de Paula FJ, Abreu DC. Postural control and functional strength in patients with type 2 diabetes mellitus with and without peripheral neuropathy. Arch Phys Med Rehabil. 2013;94(12):2465-70.

39. Uccioli L, Giacomini PG, Monticone G, Magrini A, Durola L, Bruno E, et al. Body sway in diabetic neuropathy. Diabetes Care. 1995;18(3):339-44.

40. Boucher P, Teasdale N, Courtemanche R, Bard C, Fleury M. Postural stability in diabetic polyneuropathy. Diabetes Care. 1995;18(5):638-45.

41. Turcot K, Allet L, Golay A, Hoffmeyer P, Armand S. Postural strategies in diabetes patients with peripheral neuropathy determined using cross-correlation functions. Diabetes Technol Ther. 2012;14(5):403-10.

42. Turcot K, Allet L, Golay A, Hoffmeyer P, Armand S. Investigation of standing balance in diabetic patients with and without peripheral neuropathy using accelerometers. Clin Biomech (Bristol, Avon). 2009;24(9):716-21. 
43. Chau RM, Ng TK, Kwan RL, Choi CH, Cheing GL. Risk of fall for people with diabetes. Disabil Rehabil. 2013;35(23):1975-80.

44. Kwan MM, Lin SI, Chen CH, Close JC, Lord SR. Sensorimotor function, balance abilities and pain influence Timed Up and Go performance in older community-living people. Aging Clin Exp Res. 2011;23(3):196-201.

45. DeMott TK, Richardson JK, Thies SB, Ashton-Miller JA. Falls and gait characteristics among older persons with peripheral neuropathy. Am J Phys Med Rehabil. 2007;86(2):125-32.

46. Lord SR, Delbaere K, Gandevia SC. Use of a physiological profile to document motor impairment in ageing and in clinical groups. J Physiol. 2016;594(16):4513-23.

47. Thanthrige RS, Dassanayake S, Dissanayake D, Relationship between increased risk of falling and cognitive impairment in residents of an elderly home in the Colombo district. Ceylon Med J. 2014;59(1):21-3.

Figure 1. Physiological falls risk profiles for the D-noPN (panel A) and D-PN (panel B) groups.

Each bar represents a mean Z-score for the two groups based on normative values in community-dwelling people $\geq 65$ [22]. Larger positive or negative Z-scores represent deviation from the general older population, with negative scores representing poorer performance. 
Table 1. Anthropometric, demographic and medical measures for the D-PN, D-noPN and HC groups

\begin{tabular}{lllll}
\hline Risk factor & $\mathrm{D}-\mathrm{PN}(\mathrm{n}=51)$ & $\mathrm{D}-\mathrm{noPN}(\mathrm{n}=52)$ & $\mathrm{HC}(\mathrm{n}=50)$ & $P$ value \\
\hline Demographic/Medical & & & & \\
Age (years) & $61.6 \pm 6.2$ & $61.5 \pm 5.9$ & $59.9 \pm 7.3$ & .347 \\
Height $(\mathbf{c m})$ & $156 \pm 8$ & $157 \pm 7$ & $159 \pm 8$ & .161 \\
Weight $(\mathbf{k g})$ & $60.9 \pm 10.3$ & $62.6 \pm 8.4$ & $62.4 \pm 11.4$ & .633 \\
BMI & 24.9 & 25.3 & 24.6 & .594 \\
DM duration & $17.5 \pm 7.6$ & $11.8 \pm .7 .0$ & 0 & $<.001^{* *}$ \\
Number of medications & $7.4 \pm 2.5$ & $6.3 \pm 2.67$ & $1.9 \pm 2.9$ & $<.001^{* *}$ \\
Neuropathy status & & & & $<.001^{* *}$ \\
Symptom score & $2.33 \pm 1.12$ & $.44 \pm .85$ & $.14 \pm .35$ & $<.001^{* *}$ \\
Examination score & $5.33 \pm 2.96$ & $1.04 \pm 1.33$ & $.20 \pm .49$ & \\
\hline$*$ P<0.001, *P<0.05 & & & &
\end{tabular}

Diabetes mellitus duration, number of medications, symptom and examination score were significantly different among groups. 
Table 2. Sensorimotor test performances for the D-PN, D-noPN and HC groups

\begin{tabular}{|c|c|c|c|c|c|c|c|}
\hline $\begin{array}{l}\text { Sensorimotor } \\
\text { function }\end{array}$ & $\mathrm{HC}$ & D-noPN & D-PN & F Statistic & $\begin{array}{l}\mathrm{HC} \text { vs D- } \\
\text { noPN } \\
P\end{array}$ & $\begin{array}{l}\text { HC vs. D-PN } \\
P\end{array}$ & $\begin{array}{l}\text { D-noPN vs. } \\
\text { D-PN } \\
P\end{array}$ \\
\hline $\begin{array}{l}\text { Tibial nerve } \\
\text { velocity }^{\#}\end{array}$ & $44.38 \pm 6.77$ & $40.89 \pm 4.43$ & $39.69 \pm 6.95$ & 8.49 & 0.013 & $<0.001$ & 0.392 \\
\hline $\begin{array}{l}\text { Vibration } \\
\text { perception threshold } \\
\text { (v) }\end{array}$ & $11.45 \pm 3.95$ & $16.34 \pm 7.11$ & $39.93 \pm 9.43$ & 226.98 & 0.002 & $<0.001$ & $<0.001$ \\
\hline $\begin{array}{l}\text { Proprioception } \\
\text { (degrees) }\end{array}$ & $1.54 \pm 1.35$ & $2.25 \pm 1.21$ & $2.67 \pm 1.45$ & 10.24 & 0.015 & $<0.001$ & 0.212 \\
\hline $\begin{array}{l}\text { Tactile sensitivity } \\
\text { (bending force) }\end{array}$ & $3.51 \pm .58$ & $3.80 \pm .49$ & $4.45 \pm .93$ & 23.83 & 0.039 & $<0.001$ & $<0.001$ \\
\hline $\begin{array}{l}\text { Contrast sensitivity } \\
\text { (dB) }\end{array}$ & $20.84 \pm 1.97$ & $20.62 \pm 2.12$ & $19.86 \pm 3.0$ & 2.28 & 0.885 & 0.107 & 0.256 \\
\hline $\begin{array}{l}\text { Knee extension } \\
\text { strength (kg) }\end{array}$ & $28.02 \pm 8.76$ & $24.94 \pm 6.70$ & $20.46 \pm 7.71$ & 12.14 & 0.115 & $<0.001$ & 0.011 \\
\hline $\begin{array}{l}\text { Simple reaction time } \\
(\mathrm{ms})\end{array}$ & $241.59 \pm 45.34$ & $249.32 \pm 43.12$ & $275.76 \pm 50.91$ & 7.52 & 0.679 & 0.001 & 0.013 \\
\hline $\begin{array}{l}\text { Five time sit to stand } \\
\text { test (s) }\end{array}$ & $11.14 \pm 2.21$ & $12.45 \pm 2.58$ & $14.23 \pm 3.60$ & 15.25 & 0.036 & $<0.001$ & 0.007 \\
\hline
\end{tabular}

\#n; D-PN=39, HC=52, DC=49 (tibial nerve velocity was not detected in the remainder of participants)

High scores in the vibration sense, proprioception, tactile sensitivity, reaction time and low scores in the tibial nerve, contrast sensitivity and knee extension strength assessments in D-PN group were reported compared to D-noPN and HC groups 
Table 3- Balance, mobility, fear of falling and risk of falling among the D-PN, D-noPN and HC groups

\begin{tabular}{|c|c|c|c|c|c|c|c|}
\hline $\begin{array}{l}\text { Sensorimotor } \\
\text { function }\end{array}$ & $\mathrm{HC}$ & D-noPN & D-PN & F Statistic & $\begin{array}{l}\text { HC vs D- } \\
\text { noPN } \\
P\end{array}$ & $\begin{array}{l}\text { HC vs. D-PN } \\
P\end{array}$ & $\begin{array}{l}\text { D-noPN vs. } \\
\text { D-PN } \\
P\end{array}$ \\
\hline $\begin{array}{l}\text { Sway floor, eyes } \\
\text { open-path }(\mathbf{m m})\end{array}$ & $64.02 \pm 28.55$ & $69.54 \pm 31.70$ & $74.24 \pm 33.41$ & 1.22 & 0.619 & 0.269 & 0.804 \\
\hline $\begin{array}{l}\text { Sway floor, eyes } \\
\text { closed-path (mm) }\end{array}$ & $85.66 \pm 40.54$ & $84.56 \pm 34.64$ & $106.49 \pm 54.01$ & 3.21 & 0.969 & 0.059 & 0.098 \\
\hline $\begin{array}{l}\text { Sway foam, eyes } \\
\text { opened-path }(\mathrm{mm})\end{array}$ & $159.86 \pm 99.95$ & $179.27 \pm 84.41$ & $223.35 \pm 96.42$ & 7.88 & 0.246 & $<0.001$ & 0.05 \\
\hline $\begin{array}{l}\text { Sway foam, eyes } \\
\text { closed-path (mm) }\end{array}$ & $382.84 \pm 138.19$ & $418.54 \pm 164.56$ & $491.94 \pm 188.08$ & 5.75 & 1.000 & $<0.001$ & 0.003 \\
\hline One leg stand test (s) & $28.60 \pm 3.93$ & $26.77 \pm 5.71$ & $15.93 \pm 10.11$ & 47.46 & 0.398 & $<0.001$ & $<0.001$ \\
\hline $\begin{array}{l}\text { Timed-up and go } \\
\text { test (s) }\end{array}$ & $6.51 \pm 0.96$ & $7.01 \pm 1.1$ & $8.51 \pm 2.11$ & 25.09 & 0.209 & $<0.001$ & $<0.001$ \\
\hline PPA fall risk score & $.49 \pm 0.96$ & $.74 \pm .80$ & $1.68 \pm 1.13$ & 21.98 & 0.381 & $<0.001$ & $<0.001$ \\
\hline Icon-FES score & $20.72 \pm 7.75$ & $24.65 \pm 7.2$ & $30.18 \pm 6.75$ & 21.78 & 0.019 & $<0.001$ & $<0.001$ \\
\hline
\end{tabular}

High scores in the sway tests, timed up and go test, PPA and Icon-FES assessments significantly higher in D-PN compared to D-noPN and HC group. 
